\title{
Early alkaline phosphatase dynamics as biomarker of survival in metastatic castration-resistant prostate cancer patients treated with radium-223
}

\author{
Maarten J. van der Doelen ${ }^{1,2}$ (D) $\cdot$ Agnes Stockhaus $^{3} \cdot$ Yuanjun $\mathrm{Ma}^{4} \cdot$ Niven Mehra $^{2} \cdot$ Jeffrey Yachnin ${ }^{4,5}$. \\ Winald R. Gerritsen ${ }^{2} \cdot$ Sten Nilsson ${ }^{4,5} \cdot$ Inge M. van Oort $^{1} \cdot$ Anders Ullén $^{4,5}$
}

Received: 29 January 2021 / Accepted: 23 February 2021 / Published online: 8 March 2021

(C) The Author(s) 2021

\begin{abstract}
Purpose Radium-223 is a life-prolonging therapy for castration-resistant prostate cancer (CRPC) patients with symptomatic bone metastases. However, validated biomarkers for response monitoring are lacking. The study aim was to investigate whether early alkaline phosphatase (ALP) dynamics after the first radium-223 injection can act as surrogate marker for overall survival (OS). Methods This retrospective multicenter study included consecutive CRPC patients treated with radium-223. Patients were divided into four subgroups based on baseline ALP level (normal/elevated) and early ALP response, defined as $\geq 10 \%$ ALP decrease after the first radium-223 injection. Primary endpoint was OS among the subgroups. Secondary endpoints included time to first skeletal-related event, time to ALP progression, and treatment completion rate.

Results A total of 180 patients were included for analysis. Median OS was 13.5 months (95\% confidence interval 11.5-15.5). Patients with elevated baseline ALP without ALP response after the first injection had significantly worse OS when compared to all other patients (median OS 7.9 months versus 15.7 months, hazard ratio 2.56, 95\% confidence interval 1.73-3.80, $P<0.001$ ). Multivariate analysis demonstrated that elevated baseline ALP without ALP response after the first injection, the number of prior systemic therapies, baseline LDH level, and baseline ECOG performance status were prognostic factors of OS. Patients with elevated baseline ALP without ALP response after the first injection had significantly shorter times to ALP progression and first skeletal-related event, and more frequently discontinued radium-223 therapy when compared to other patients.

Conclusion Early treatment-induced changes in ALP after one radium-223 injection were associated with OS in metastatic CRPC patients.
\end{abstract}

Keywords Alkaline phosphatase $\cdot$ Biomarker $\cdot$ Bone metastases $\cdot$ Castration-resistant prostate cancer $\cdot$ Radium-223 $\cdot$ Prognostic variables

This article is part of the Topical Collection on Oncology - Genitourinary

Maarten J. van der Doelen

Maarten.vanderDoelen@ radboudumc.nl

1 Department of Urology, Radboud University Medical Center, Geert Grooteplein Zuid 10, 6525, GA Nijmegen, The Netherlands

2 Department of Medical Oncology, Radboud University Medical Center, Nijmegen, The Netherlands

3 Department of Oncology-Pathology, Mälarsjukhuset, Eskilstuna, Sweden

4 Department of Oncology-Pathology, Karolinska Institutet, Stockholm, Sweden

5 Department of Pelvic Cancer, Genitourinary Oncology and Urology Unit, Karolinska University Hospital, Stockholm, Sweden

\section{Introduction}

Radium-223 is an alpha emitter that selectively binds to areas of increased bone turnover in bone metastases and emits highenergy alpha particles of short range, causing double-strand DNA breaks [1]. Based on significant survival benefit in the pivotal phase 3 ALSYMPCA trial, radium-223 is a registered treatment option for castration-resistant prostate cancer (CRPC) patients with symptomatic bone metastases and without any known visceral metastases [2].

Approximately $90 \%$ of CRPC patients eventually develop bone metastases $[3,4]$. In these patients, serum total alkaline phosphatase (ALP) levels are often elevated and, in the absence of extensive liver disease, these levels reflect osteoblastic activity and the extent of disease [5]. This is of clinical 
importance, since elevated ALP levels are associated with the occurrence of skeletal-related events (SREs), independent of therapy $[6,7]$. In addition, several retrospective series have reported that elevated baseline ALP levels were associated with inferior overall survival $(\mathrm{OS})$ in patients treated with radium-223 [8-11].

In treatment responders, ALP has previously been shown to decrease already 4 weeks after initiation of radium-233 and may continue to decrease until the end of treatment [9]. Although the implication of baseline ALP levels on outcome has been studied, studies on ALP dynamics during and after radium-223 treatment are limited, and the utility of changes in ALP as a biomarker has to be explored [5]. In the ALSYMPCA trial, $47 \%$ of the patients experienced $\geq 30 \%$ ALP reduction during therapy, and real-world studies have described $\geq 25 \%$ ALP decrease in $50-62 \%$ of patients after initiation of radium-223 treatment [10, 12-14]. Post hoc analyses from the ALSYMPCA trial suggested that an ALP response of $>30 \%$ at week 12 of therapy is associated with prolonged survival and longer time to first symptomatic skeletal event $[9,15]$. Similar findings have been described in two small retrospective series of patients treated with radium-223, where OS was found to be significantly longer for patients with $\geq 30 \%$ ALP reduction during therapy when compared to ALP nonresponders $[10,14]$. However, there are currently no early biomarkers to predict overall survival during treatment with radium-223 in daily practice. The aim of this study was to investigate the prognostic value of early ALP dynamics during radium-223 therapy in patients with metastatic CRPC.

\section{Methods}

\section{Study design, setting, and participants}

This was a multicenter retrospective cohort study including consecutive patients treated with standard dosing of radium223 at Karolinska University Hospital between October 2012 and December 2016, and at Radboud University Medical Center between September 2013 and January 2018. All patients were treated with radium-223 according to the original label, prior to the EMA label change of July 2018. The study was approved by the local medical ethics committees and both hospital review boards. The regulations of the Helsinki declaration were followed. Inclusion criteria were histological confirmed prostate cancer, CRPC as defined by the European Association of Urology guidelines, radiological evidence of bone metastases on bone scintigraphy, and at least one administered injection of radium-223 [16]. Patients who were concomitantly treated with abiraterone or enzalutamide were excluded.

\section{Data collection and follow-up}

The medical records of patients were reviewed to collect demographics and clinical characteristics, including Gleason score and tumor staging, treatments and SREs prior to radium-223 therapy, use of analgesics at baseline, baseline Eastern Cooperative Oncology Group (ECOG) performance status, the use of bone health agents (denosumab or bisphosphonates) during treatment with radium-223, the number of radium-223 injections, biochemical parameters (ALP, lactate dehydrogenase (LDH), and prostate-specific antigen (PSA)), hematological parameters (hemoglobin and platelet count), date of first SRE after radium-223 initiation, and date of death. All patients were followed until death or November $1,2020$.

\section{Subgroup categorization}

Elevated ALP was defined as baseline ALP level above the upper limit of normal of $115 \mathrm{U} / \mathrm{L}$ (1.9 ukat/L), according to institutional criteria. ALP response from baseline was defined as $\geq 10 \%$ ALP decline from baseline, measured 3-4 weeks after the first radium-223 injection. Non-response was classified as an ALP change of $<10 \%$ or no decrease from baseline after the first radium-223 injection. The 10\% ALP response cut-off was chosen because a less pronounced ALP response at an earlier time point was expected when compared to the $\geq 30 \%$ ALP reduction outcome measure at week 12 of therapy that was applied in the ALSYMPCA trial [2]. Moreover, the $10 \%$ ALP cut-off was used previously in a retrospective cohort study by Dizdarevic et al. [10]. Based on baseline ALP levels and ALP response from baseline, the cohort was divided in four pre-specified subgroups. Subgroups 1 and 2 consisted of patients who had normal baseline ALP levels, with and without ALP response, respectively. Similarly, subgroups 3 and 4 consisted of patients with elevated baseline ALP levels, with and without subsequent $\geq 10 \%$ ALP decline after the first radium-223 injection, respectively.

\section{Study endpoints}

The primary study endpoint was OS. OS was defined as time from first radium-223 injection until death from any cause or last recorded date of follow-up. We analyzed OS in the predefined subgroups and evaluated prognostic factors associated with OS. Secondary endpoints included biomarker dynamics before, during, and after radium-223 therapy, the time to ALP progression, the time to first SRE, and the number of administered radium-223 injections.

ALP levels were captured 12, 9, 6, and 3 months prior to radium-223 treatment, every 4 weeks during radium-223 treatment and 3, 6, 9, and 12 months after the last radium-223 injection. Likewise, PSA levels were recorded during and 
after radium-223. In addition to ALP response of $10 \%$ after the first radium-223 injection, we also evaluated changes in PSA and ALP, calculated as maximal decline from baseline during radium-223 therapy, with $30 \%$ cut-off, according to the ALSYMPCA study definition [2]. Normalization of ALP was defined as an ALP level below $115 \mathrm{U} / \mathrm{L}$ in patients with elevated ALP levels at baseline. ALP progression was defined as an increase of $\geq 25 \%$ from baseline in patients with no decrease from baseline, or as an increase of $\geq 25 \%$ above the nadir, according to the ALSYMPCA study criteria [2]. In case a next systemic therapy was started prior to $\geq 25 \%$ increase of ALP above the nadir, no ALP progression was documented and the patient was censored. SREs were defined as surgery or radiotherapy to the bone, spinal cord compression, and pathological fractures, according to Prostate Cancer Working Group 3 criteria [17].

\section{Statistical analysis}

Descriptive statistical methods were used to characterize the cohort. To compare the subgroups, the chi-square and MannWhitney $U$ tests were used for categorial variables and continuous variables, respectively. Kaplan-Meier statistics were used to calculate time-to-event data. Univariate and multivariate Cox proportional hazard regression models were used to compare time-to-event distributions between the pre-specified subgroups and to assess the prognostic significance of baseline variables, presented as hazard ratios (HRs) with 95\% confidence intervals (CIs). In multivariate models, forward selection and backward elimination to add or remove covariates were used. We adjusted for the following covariates at the start of radium-223 treatment: age and Gleason score at diagnosis, time from development of CRPC to initiation of radium-223 therapy, number of therapies before radium-223 initiation, ECOG performance status, and baseline hemoglobin, LDH, and PSA levels. The covariates LDH and PSA were $\log$ transformed due to distribution skewness. All statistical tests were two-sided, with $P$ values of $<0.05$ considered to be statistically significant.

\section{Results}

\section{Patient cohort}

In total, 197 patients with metastatic CRPC were treated at both centers during the study period. Seventeen patients were excluded from further analysis due to concomitant therapies or missing data (Fig. 1). Therefore, 180 patients were included for analysis. Baseline demographics and clinical characteristics of the total cohort and patient subgroups are shown in Table 1. Patients received a median of two prior systemic therapies, in addition to androgen deprivation therapy, for
CRPC. One hundred twenty-eight (71\%) patients were previously treated with abiraterone or enzalutamide and 113 (63\%) patients underwent prior taxane-based chemotherapy, either upfront in hormone-sensitive (4\%) or in castration-resistant $(96 \%)$ state. Ninety (50\%) patients had a prior SRE and bone health agents were given prior to or during radium-223 therapy in half of the patient cohort. Although bone health agents were more frequently used in patients with normal baseline ALP levels (56\%) when compared to patients with high baseline ALP levels (46\%), there was no statistically significant difference between the subgroups $(P=0.193)$.

The median ALP level at baseline was $156 \mathrm{U} / \mathrm{L}$ (interquartile range 95-264). Overall, 67 (37\%) patients had normal ALP levels prior to treatment and $113(63 \%)$ patients had elevated baseline ALP levels. Data on ALP dynamics after the first injection were missing in eight (4\%) patients. Hence, data on ALP dynamics were available for analysis in 66 patients with normal baseline ALP levels and 106 patients with elevated baseline ALP levels. One hundred twelve (62\%) patients experienced $\geq 10 \%$ ALP decrease after the first radium-223 injection. Subgroups 1, 2, 3, and 4 included 39, 27, 73, and 33 patients, respectively (Fig. 1). The patients in subgroup 4 were significantly younger at time of radium-223 initiation, used opioids more frequently, were more heavily pretreated, and had significantly lower baseline hemoglobin levels and higher LDH levels (Table 1).

\section{ALP dynamics and overall survival}

At time of analysis, $172(96 \%)$ patients had deceased. The median follow-up was 13.6 (range 1-79) months. The median OS for the total study population was 13.5 months $(95 \% \mathrm{CI}$ 11.5-15.5). OS data for the total cohort and subgroups are shown in Table 2. Patients with normal baseline ALP levels (subgroups 1-2) had significantly longer OS when compared to patients with elevated baseline ALP levels (subgroups 3-4; median OS 19.5 months versus 10.8 months, HR 2.08, 95\% CI $1.51-2.86, P<0.001$, Fig. 2a). Furthermore, patients with elevated baseline ALP without $\geq 10 \%$ ALP decline after the first injection (subgroup 4) had significantly worse OS than the patients in subgroups 1-3 (median OS 7.9 months versus 15.7 months, HR $2.56,95 \%$ CI 1.73-3.80, $P<0.001$, Fig. 2b). This association remained statistically significant after adjusting for baseline covariates (HR 2.15, 95\% CI 1.353.43). The final multivariate model selected subgroup 4 , baseline LDH level, baseline ECOG performance status, and the number of prior systemic therapies as prognostic factors of OS (Table 3).

\section{Biomarker dynamics}

Of the 174 patients with available data on ALP response during therapy, $108(62 \%)$ patients experienced $\geq 30 \%$ ALP 


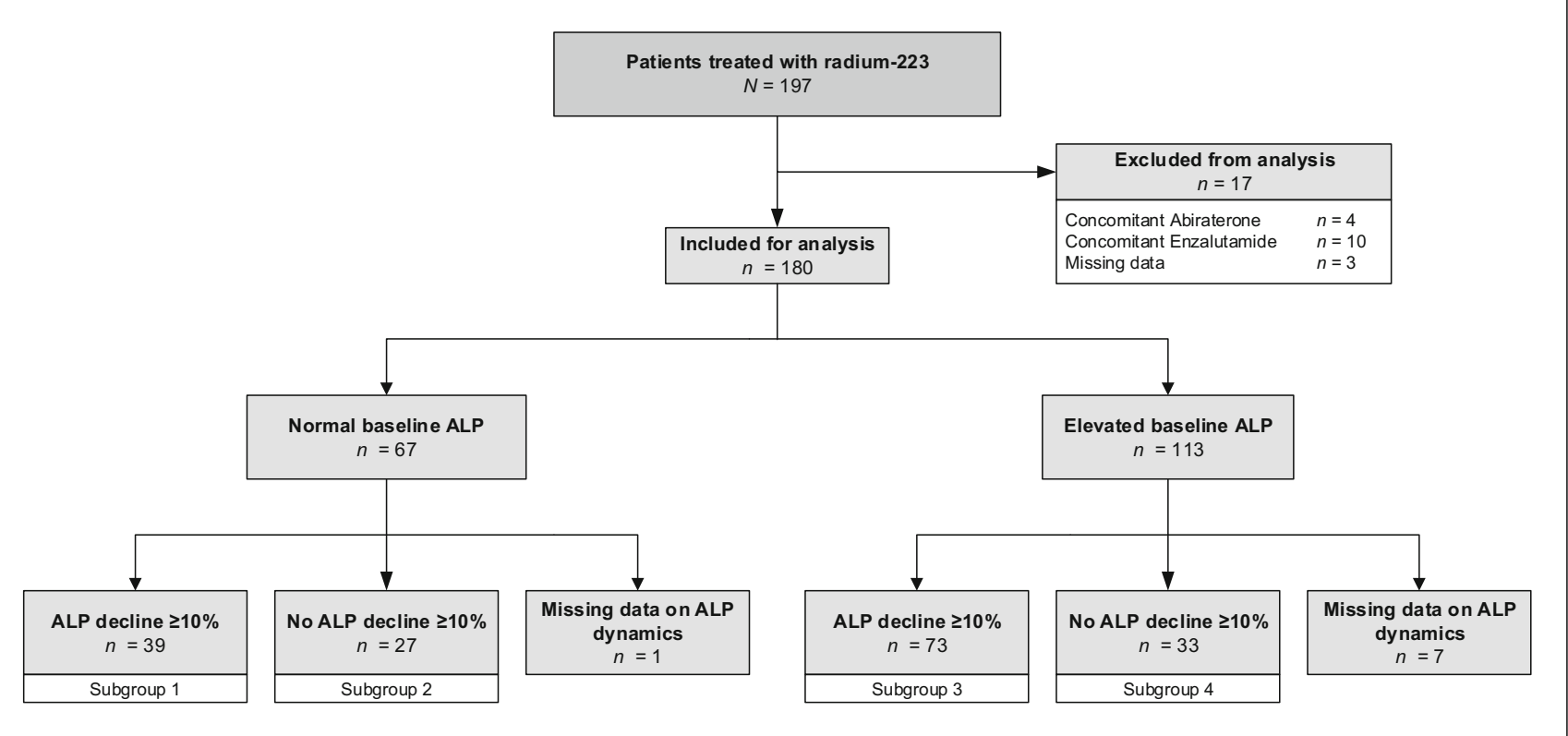

Fig. 1 Consort diagram of the study population, including subgroup categorization based on ALP dynamics after the first injection of radium-223

decrease during radium-223 therapy. Forty-six (27\%) patients already showed $\geq 30 \%$ ALP decrease after the first radium- 223 injection. The response definition of $\geq 10 \%$ ALP decrease after the first injection correlated with the observed $\geq 30 \%$ ALP decrease throughout therapy in $80 \%$ of the patients. Out of 107 patients with elevated baseline ALP levels and available data on ALP dynamics, normalization of ALP during therapy occurred in $51(48 \%)$ patients. Figure 3 a shows the change in ALP level at $12,9,6$, and 3 months before radium-223, at each injection of radium-223 and 3, 6, 9, and 12 months after radium-223.

One hundred forty-four $(80 \%)$ patients developed ALP progression before initiation of a subsequent systemic therapy. The median time to ALP progression was 5.7 months (95\% CI 5.0-6.3). Patients in subgroup 4 had significantly shorter time to ALP progression when compared to all other patients (2.5 months versus 5.7 months, HR 2.30, 95\% CI 1.493.56, $P<0.001$, Fig. $2 \mathrm{c})$.

In the cohort, the median PSA level at baseline was $166 \mu \mathrm{g} / \mathrm{L}$ (interquartile range $59-417$ ). A $30 \%$ or greater reduction in PSA level during therapy was achieved in $29(17 \%)$ patients. Of these PSA responding patients, 21 (72\%) patients had $\geq 10 \%$ ALP decrease after the first radium-223 injection and $25(86 \%)$ patients achieved $\geq 30 \%$ ALP decrease during radium-223 therapy. Figure $3 \mathrm{~b}$ shows the change in PSA level at each radium-223 injection and 3, 6, 9, and 12 months after the last radium-223 injection.

\section{Treatment completion}

Overall, the median number of administered injections was six and $110(61 \%)$ patients completed radium-223 therapy. Only $39 \%$ of the patients in subgroup 4 had completed radium-223 therapy, compared to $68 \%$ of the patients in the other subgroups. The median number of administered injections was six in subgroups $1-3$ and four in subgroup $4(P<0.001)$. Of the eight patients with missing data on ALP dynamics, four (50\%) patients received just one radium-223 injection.

\section{Skeletal-related events}

During or after radium-223, $106(59 \%)$ patients experienced at least one SRE. In 51 (48\%) of these patients, an SRE occurred within 6 months after initiation of radium-223 therapy. The median time to the first SRE was 12.0 months (95\% CI 8.315.7). Patients in subgroup 4 had a significantly shorter time to occurrence of the first SRE when compared to all other patients (5.6 months versus 14.6 months, HR 2.01, 95\% CI 1.27-3.32, $P=0.003$, Fig. 2 d). In patients with an elevated baseline ALP, the median time to SRE was significantly longer when patients experienced $\geq 10 \%$ ALP decline after the first injection (subgroup 3) when compared to the patients without such ALP reduction (subgroup 4) (14.4 months versus 5.6 months, HR 1.82, 95\% CI 1.07-3.09, $P=0.027)$.

\section{Discussion}

In this retrospective study of a real-world population of CRPC patients with bone metastases, we demonstrate that patients with elevated baseline ALP without subsequent $\geq 10 \%$ ALP decline after the first injection had significantly worse OS when compared to patients with ALP response after the first radium-223 injection. Our results are in line with a previous smaller retrospective study that showed overall survival was 
Table 1 Baseline patient demographics and clinical characteristics

\begin{tabular}{|c|c|c|c|c|c|c|c|c|c|c|}
\hline \multirow[b]{3}{*}{ Age at start of radium-223 [y] } & \multicolumn{3}{|c|}{ Complete cohort $(N=180)$} & \multicolumn{3}{|c|}{ Subgroups 1-3 $(N=139)$} & \multicolumn{3}{|c|}{ Subgroup $4(N=33)$} & \multirow{3}{*}{$\begin{array}{l}P \text { value } \\
\mathbf{0 . 0 3 0}\end{array}$} \\
\hline & \multirow{2}{*}{$\frac{n}{180}$} & \multicolumn{2}{|c|}{ Median [IQR] or $n(\%)$} & \multirow{2}{*}{$\frac{n}{139}$} & \multicolumn{2}{|c|}{ Median [IQR] or $n(\%)$} & \multirow{2}{*}{$\frac{n}{33}$} & \multicolumn{2}{|c|}{ Median [IQR] or $n(\%)$} & \\
\hline & & 71 & {$[66-76]$} & & 71 & {$[67-76]$} & & 69 & {$[62-73]$} & \\
\hline \multicolumn{11}{|l|}{ Initial tumor staging } \\
\hline Localized prostate cancer & 180 & 90 & $(50.0)$ & 139 & 70 & $(50.4)$ & 33 & 13 & $(39.4)$ & \multirow{2}{*}{0.257} \\
\hline Metastatic prostate cancer & 180 & 90 & $(50.0)$ & 139 & 69 & $(49.6)$ & 33 & 20 & $(60.6)$ & \\
\hline ISUP 1 (Gleason score $\leq 6)$ & 148 & 10 & $(6.8)$ & 113 & 9 & $(8.0)$ & 30 & 1 & (3.3) & \multirow[t]{3}{*}{0.567} \\
\hline ISUP 2-3 (Gleason score 7) & 148 & 40 & $(27.0)$ & 113 & 30 & $(26.5)$ & 30 & 10 & $(33.3)$ & \\
\hline ISUP 4-5 (Gleason score $\geq 8$ ) & 148 & 98 & $(66.2)$ & 113 & 74 & $(65.5)$ & 30 & 19 & $(63.3)$ & \\
\hline Time CRPC to radium-223 [mo] & 180 & 23.2 & {$[12.0-37.9]$} & 139 & 25.3 & {$[12.0-38.0]$} & 33 & 21.0 & {$[10.7-35.0]$} & 0.323 \\
\hline \multicolumn{11}{|l|}{ Prior registered therapies for CRPC } \\
\hline Median number of therapies & 180 & 2 & {$[1-3]$} & 139 & 2 & {$[1,2]$} & 33 & 2 & {$[1-3]$} & 0.031 \\
\hline None & 180 & 35 & $(19.4)$ & 139 & 29 & $(20.9)$ & 33 & 4 & $(12.1)$ & 0.329 \\
\hline Docetaxel $^{1}$ & 180 & 109 & $(60.6)$ & 139 & 82 & $(59.0)$ & 33 & 24 & $(72.7)$ & 0.145 \\
\hline Cabazitaxel $^{2}$ & 180 & 46 & $(25.6)$ & 139 & 30 & $(21.6)$ & 33 & 12 & $(36.4)$ & 0.076 \\
\hline Abiraterone & 180 & 95 & $(52.8)$ & 139 & 75 & $(54.0)$ & 33 & 16 & $(48.5)$ & 0.571 \\
\hline Enzalutamide & 180 & 52 & $(28.9)$ & 139 & 31 & $(22.3)$ & 33 & 18 & $(54.5)$ & $<0.001$ \\
\hline Opioid use & 180 & 79 & $(43.9)$ & 139 & 52 & $(37.4)$ & 33 & 21 & $(63.6)$ & 0.006 \\
\hline Prior SRE & 180 & 90 & $(50.0)$ & 139 & 72 & $(51.8)$ & 33 & 14 & $(42.4)$ & 0.333 \\
\hline \multicolumn{11}{|l|}{ Bone health agent use } \\
\hline None & 177 & 89 & $(50.3)$ & 136 & 68 & $(50.0)$ & 33 & 17 & $(51.5)$ & \multirow[t]{3}{*}{0.859} \\
\hline Denosumab & 177 & 48 & $(27.1)$ & 136 & 39 & $(28.7)$ & 33 & 8 & $(24.2)$ & \\
\hline Bisphosphonates & 177 & 40 & $(22.6)$ & 136 & 29 & $(21.3)$ & 33 & 8 & $(24.2)$ & \\
\hline \multicolumn{11}{|l|}{ ECOG performance status } \\
\hline ECOG 0 & 172 & 91 & $(52.9)$ & 134 & 74 & $(55.2)$ & 31 & 15 & $(48.4)$ & \multirow[t]{3}{*}{0.784} \\
\hline ECOG 1 & 172 & 64 & $(37.2)$ & 134 & 48 & $(35.8)$ & 31 & 13 & $(41.9)$ & \\
\hline ECOG 2-3 & 172 & 17 & $(9.9)$ & 134 & 12 & $(9.0)$ & 31 & 3 & $(9.7)$ & \\
\hline Hemoglobin (g/dL) & 180 & 12.3 & {$[11.4-13.2]$} & 139 & 12.6 & {$[11.6-13.4]$} & 33 & 11.8 & {$[10.5-12.6]$} & 0.001 \\
\hline $\mathrm{Hb} \geq 10$ & 180 & 168 & $(93.3)$ & 139 & 133 & $(95.7)$ & 33 & 28 & $(84.8)$ & \multirow[t]{2}{*}{0.022} \\
\hline $\mathrm{Hb}<10$ & 180 & 12 & $(6.7)$ & 139 & 6 & $(4.3)$ & 33 & 5 & $(15.2)$ & \\
\hline Platelet count $\left(\times 10^{9} / \mathrm{L}\right)$ & 180 & 244 & [206-289] & 139 & 249 & [208-289] & 33 & 241 & [198-295] & 0.616 \\
\hline PSA level ( $\mu \mathrm{g} / \mathrm{L})$ & 179 & 166 & {$[59-417]$} & 139 & 130 & {$\left[\begin{array}{ll}54 & 400\end{array}\right]$} & 32 & 249 & [82-499] & 0.125 \\
\hline ALP level (U/L) & 180 & 156 & [95-264] & 139 & 125 & [85-228] & 33 & 216 & [145-348] & $<0.001$ \\
\hline $\mathrm{ALP}<115$ & 180 & 67 & $(37.2)$ & 139 & 66 & $(47.5)$ & 33 & 0 & $(0.0)$ & \multirow[t]{2}{*}{$<0.001$} \\
\hline $\mathrm{ALP} \geq 115$ & 180 & 113 & $(62.8)$ & 139 & 73 & $(52.5)$ & 33 & 33 & $(100.0)$ & \\
\hline LD level (U/L) & 166 & 233 & [198-302] & 129 & 219 & [198-282] & 29 & 274 & [210-392] & 0.013 \\
\hline $\mathrm{LD}<250$ & 166 & 96 & $(57.8)$ & 129 & 82 & $(63.6)$ & 29 & 12 & $(41.1)$ & \multirow[t]{2}{*}{0.028} \\
\hline $\mathrm{LD} \geq 250$ & 166 & 70 & $(42.2)$ & 129 & 47 & (36.4) & 29 & 17 & $(58.6)$ & \\
\hline
\end{tabular}

${ }^{1}$ Docetaxel was administered as upfront chemotherapy in hormone-sensitive prostate cancer in four patients $(2.2 \%)$

${ }^{2}$ Four patients $(2.2 \%)$ received cabazitaxel chemotherapy without prior docetaxel chemotherapy

$A L P$, alkaline phosphatase; $C R P C$, castration-resistant prostate cancer; $E C O G$, Eastern Cooperative Oncology Group; IQR, interquartile range; ISUP, International Society of Urological Pathology; $L D$, lactate dehydrogenase; mo, months; PSA, prostate-specific antigen; SRE, skeletal related event; $y$, years

Bold $=p$ value $<0.05$

significantly longer in patients with $\geq 10 \%$ ALP reduction during therapy when compared to ALP non-responders [10]. In the present study, $84 \%$ of the patients experienced an ALP decline after the first injection, with a mean percentage change in ALP from baseline of $-11 \%$. Sixty-two percent of the patients showed $\geq 10 \%$ ALP decline after the first injection in this study. A post hoc analysis of the ALSYMPCA trial demonstrated a mean percentage change in ALP of $-20 \%$ from baseline after the first radium-223 injection [9]. However, this post hoc analysis did not investigate an association of ALP change after the first injection with response metrics and OS. 
Table 2 Overall survival data for the total cohort and the pre-specified subgroups

\begin{tabular}{|c|c|c|c|c|c|}
\hline & & $N(\%)$ & $\begin{array}{l}\text { Median survival } \\
\text { (months) }\end{array}$ & $95 \% \mathrm{CI}$ & $P$ value \\
\hline \multicolumn{2}{|c|}{ Total cohort } & $180(100)$ & 13.5 & $11.5-15.5$ & \\
\hline \multicolumn{5}{|c|}{ Subgroup } & \multirow[t]{6}{*}{$<0.001$} \\
\hline 1 & Normal baseline ALP, $\geq 10 \%$ decline after first injection & $39(21.7)$ & 25.4 & $13.4-37.3$ & \\
\hline 2 & Normal baseline ALP, $<10 \%$ decline after first injection & $27(15.0)$ & 18.2 & $11.4-24.9$ & \\
\hline 3 & Elevated baseline ALP, $\geq 10 \%$ decline after first injection & $73(40.6)$ & 12.9 & $10.5-15.3$ & \\
\hline \multirow[t]{2}{*}{4} & Elevated baseline ALP, $<10 \%$ decline after first injection & $33(18.3)$ & 7.9 & $5.9-9.9$ & \\
\hline & Missing ALP dynamics & $8(4.4)$ & 4.2 & $2.2-6.2$ & \\
\hline \multicolumn{2}{|c|}{ Subgroups $1-2$} & $66(36.7)$ & 19.5 & $11.8-27.1$ & \multirow[t]{2}{*}{$<0.001$} \\
\hline \multicolumn{2}{|c|}{ Subgroups 3-4 } & $106(58.9)$ & 10.8 & $8.2-13.4$ & \\
\hline \multicolumn{2}{|c|}{ Subgroups $1-3$} & $139(77.2)$ & 15.7 & $13.0-18.4$ & \multirow[t]{2}{*}{$<0.001$} \\
\hline \multicolumn{2}{|c|}{ Subgroup 4} & $33(18.3)$ & 7.9 & $5.9-9.9$ & \\
\hline
\end{tabular}

$A L P$, alkaline phosphatase; $C I$, confidence interval

Bold $=p$ value $<0.05$

We found that patients with normal baseline ALP levels had significantly better OS than patients with elevated baseline ALP levels. This finding correlates with previous studies that

Table 3 Multivariable Cox proportional hazard analysis of overall survival

\begin{tabular}{llll}
\hline & HR & $95 \%$ CI & $P$ value \\
\hline Age & 0.99 & $0.97-1.02$ & 0.65 \\
Time CRPC to start radium-223 & 0.99 & $0.98-1.00$ & 0.08 \\
& & & \\
$\quad$ Initial tumor Gleason score & & & \\
Gleason score $\leq 7$ & Ref & & \\
Gleason score 8-10 & 0.71 & $0.47-1.07$ & 0.10 \\
Baseline hemoglobin & 0.89 & $0.76-1.04$ & 0.15 \\
Baseline PSA (log transformed) & 1.08 & $0.97-1.21$ & 0.17 \\
Baseline LDH (log transformed) & 1.80 & $1.17-2.77$ & $<\mathbf{0 . 0 1}$
\end{tabular}

ALP dynamics during radium-223

\begin{tabular}{|c|c|c|c|}
\hline Subgroups $1-3$ & Ref & & \\
\hline Subgroup 4 & 2.15 & $1.35-3.43$ & $<0.01$ \\
\hline \multicolumn{4}{|c|}{ Baseline ECOG performance status } \\
\hline $0-1$ & Ref & & \\
\hline$\geq 2$ & 3.85 & $1.94-7.64$ & $<0.01$ \\
\hline \multicolumn{4}{|c|}{ Number of prior CRPC therapies } \\
\hline 0 & Ref & & \\
\hline$\geq 1$ & 2.46 & $1.45-4.17$ & $<0.01$ \\
\hline
\end{tabular}

$A L P$, alkaline phosphastase; $C I$, confidence interval; $C R P C$, castrationresistant prostate cancer; $E C O G$, Eastern Cooperative Oncology Group; $H R$, hazard ratio; $L D H$, lactate dehydrogenase; $P S A$, prostate specific antigen; Ref, reference

Bold $=p$ value $<0.05$ identified baseline ALP as an important prognostic factor of OS in radium-223-treated patients as well as in CRPC patients treated with other life-prolonging agents such as abiraterone and docetaxel chemotherapy $[7,9,11,18,19]$. In addition, low baseline ALP levels have also been associated with more frequent completion of radium-223 therapy [13, 20, 21]. These study outcomes highlight the prognostic value of baseline ALP measurements in the setting of metastatic CRPC.

Our findings suggest that ALP can serve as an early biomarker for treatment benefit, especially in patients with elevated baseline ALP levels. Based on early ALP changes, clinicians may be able to identify patients who need more close monitoring of clinical response during radium-223 therapy, since the absence of $\geq 10 \%$ ALP reduction may be an early indicator of treatment resistance. A post hoc analysis on data from the ALSYMPCA trial revealed that dynamic changes in ALP and lactate dehydrogenase may be useful for monitoring during treatment with radium-223, but these markers are deemed inadequate as surrogates for survival [9]. For this reason, it is not recommended to discontinue therapy solely based on changes in ALP, and other indicators of disease progression must be involved in clinical decision-making. A combinatory set of variables might have higher prognostic value. Of interest, a recent post hoc analysis of an international early access program that included 696 patients identified three risk groups based on changes in ALP and hemoglobin after three radium-223 injections. In that study, patients with ALP increase and hemoglobin decrease had significantly shorter survival than those with ALP decrease and hemoglobin increase during therapy [21].

Beyond early ALP dynamics, our multivariate analysis identified other prognostic variables of OS, including the number of prior systemic therapies and the LDH level and ECOG performance status at baseline. The prognostic value 
a

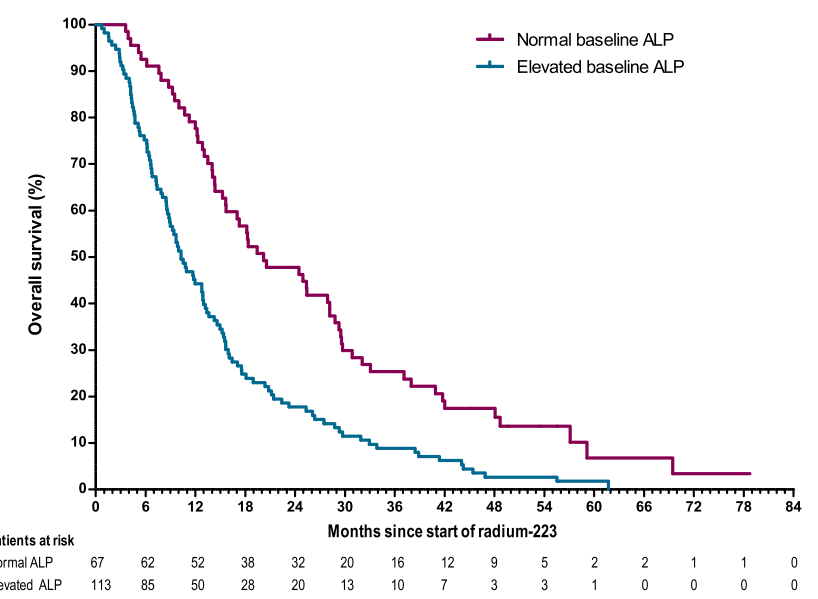

C

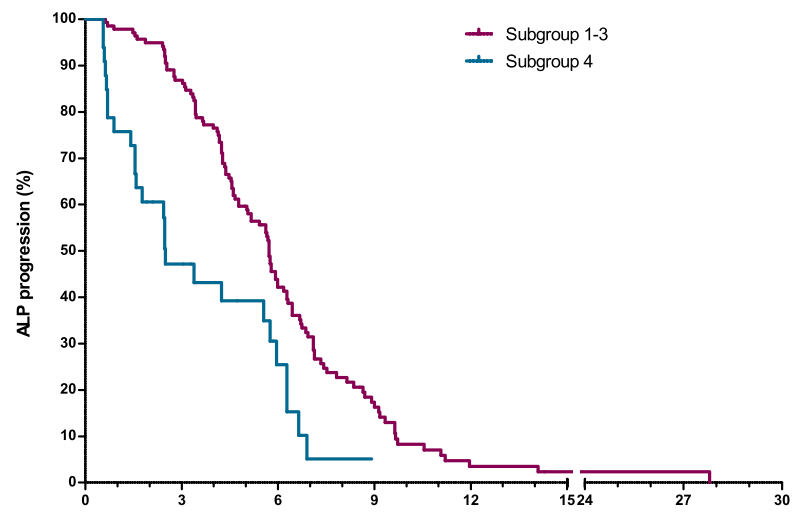

Patients at risk

Subgroup 1-3 139

Subgroup $4 \quad 33$

Fig. 2 Kaplan-Meier curves. a Overall survival, comparing the patients with normal baseline ALP levels (subgroups 1-2) to patients with elevated baseline ALP levels (subgroups 3-4). b Overall survival, comparing the patients in subgroups $1-3$ to the patients with elevated baseline ALP without $\geq 10 \%$ ALP decline after the first injection (subgroup 4). c Time to ALP progression, comparing the patients in subgroup

of the number of prior lines of therapies is of importance, since the European Medicines Agency recommended in July 2018 to restrict the use of radium-223 to patients who have had at least two previous treatments for metastatic CRPC or who cannot receive other treatments. This label change has impact on the treatment arsenal of metastatic CRPC patients. Due to the development of visceral metastases, impaired performance status and reduced hematological function in later disease stages, the window of opportunity to receive radium-223 therapy might be missed [4, 22, 23].

We found a significantly shorter time to the first SRE in patients with elevated baseline ALP without subsequent $\geq 10 \%$ ALP decline after the first injection when compared to other subjects. Of importance, bone health agents were b



d

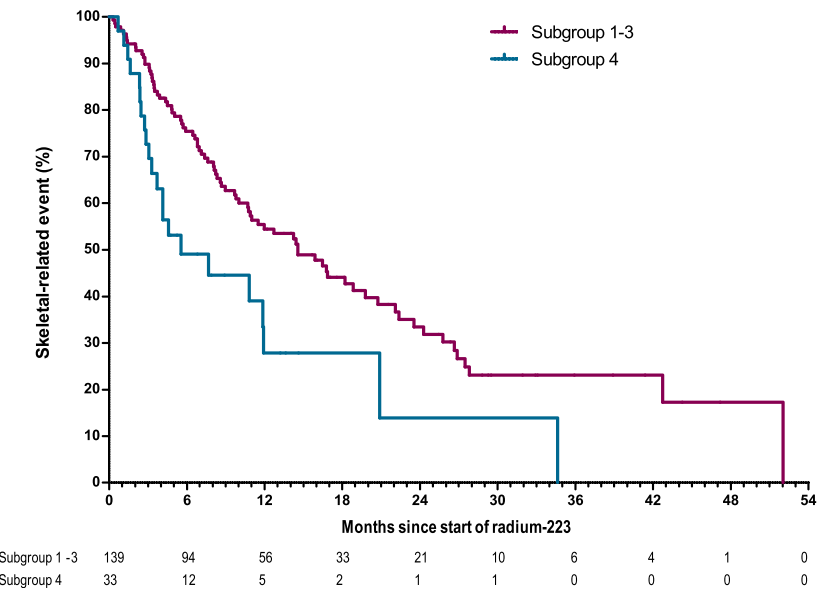

$1-3$ to patients with elevated baseline ALP without $\geq 10 \%$ ALP decline after the first injection (subgroup 4). d Time to first skeletal-related event, comparing the patients in subgroup 1-3 to patients with elevated baseline ALP without $\geq 10 \%$ ALP decline after the first injection (subgroup 4)

underutilized in the present study, with only $50 \%$ of patients receiving bisphosphonates or denosumab. However, this is comparable to the use of these agents in the prospective ALSYMPCA and REASSURE studies [2, 24]. The ALSYMPCA trial has shown that radium-223 therapy was associated with a delayed time to first symptomatic SRE when compared to placebo [15]. Since the randomized phase 3 ERA-223 trial revealed that the combination of abiraterone and prednisone plus radium-223 increased the risk of bone fractures when compared with the abiraterone and prednisone plus placebo group, the prevention of SREs in metastatic CRPC patients has gained more attention [22, 25, 26]. Our findings and the outcomes of the ERA-223 trial warrant the introduction of bone health agents to prevent skeletal 

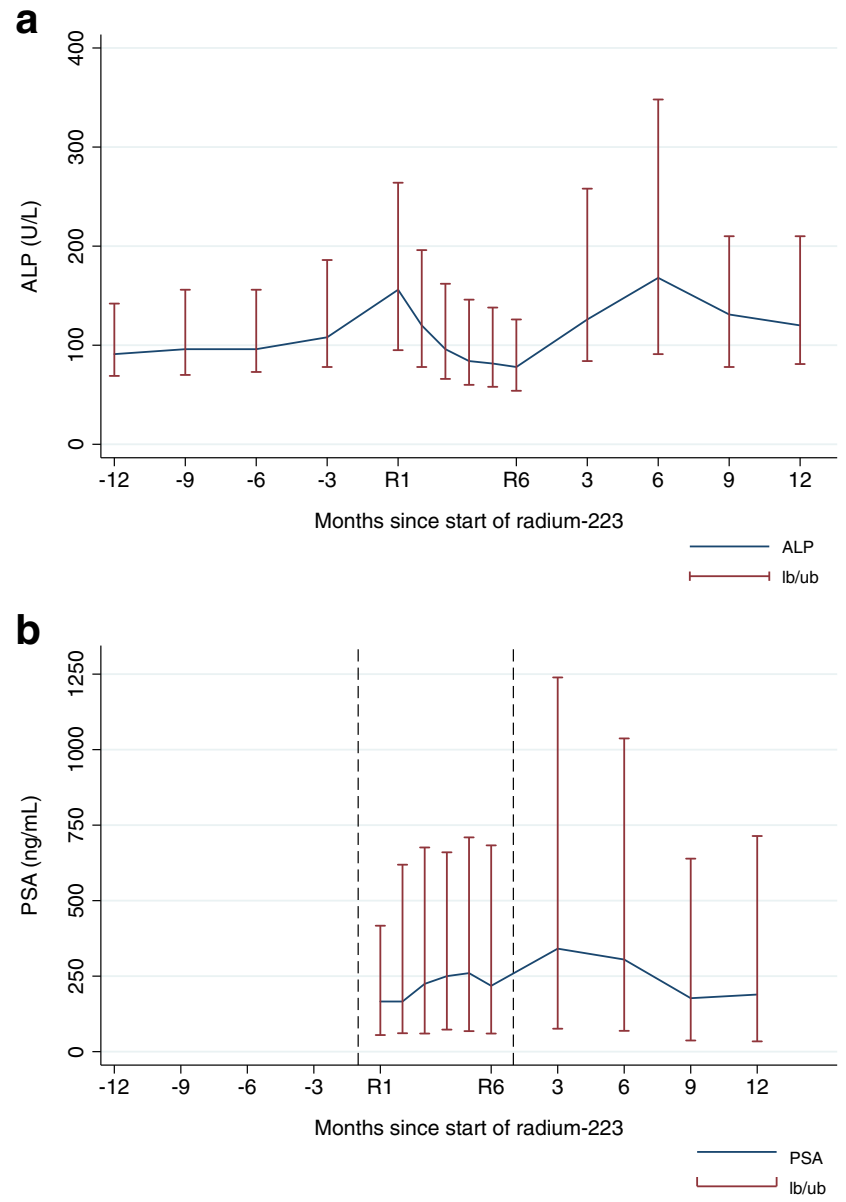

Fig. 3 Biomarker dynamics. a ALP dynamics before, during, and after treatment with radium-223 (median, interquartile range). b PSA dynamics during and after treatment with radium-223 (median, interquartile range). R1; radium-223 injection 1; R6 radium-223 injection 6

morbidity in patients with metastatic CRPC prior to initiation of radium-223, especially in patients with elevated baseline ALP levels, if not already started in an earlier phase of CRPC.

Our findings and the observations in previous studies indicate that bone-related parameters are strong prognostic variables for OS in CRPC with bone metastases. To date, validated liquid blood or urinary markers for monitoring of radium223 treatment in metastatic CRPC represent an unmet medical need. Several markers of bone metabolism, including bonespecific ALP, procollagen type $1 \mathrm{~N}$-terminal propeptide, procollagen type $1 \mathrm{C}$-terminal propeptide, $\mathrm{C}$-telopeptide of type 1 collagen, and $\mathrm{N}$-telopeptide of type 1 collagen, have been suggested as potential surrogate markers to monitor treatment with radium-223 [27-29]. Future prospective clinical studies might incorporate markers of bone turnover when evaluating radium-223 treatment.

The current study has several limitations that should be notified. Due to the retrospective nature, the data should be interpreted with caution when making decisions regarding treatment discontinuation. Selection bias might be introduced due to the exclusion of patients without follow-up ALP levels available. The missing of these data can possibly be explained by early discontinuation of radium-223 therapy due to progressive disease. Since the patients in this study were treated in two large academic hospitals, the results may differ from outcomes of patients treated in community hospitals. In addition, the majority of patients were treated in the era prior to the introduction of upfront docetaxel or abiraterone in the setting of metastatic hormone-sensitive prostate cancer. However, the study is strengthened by the multicenter design and the relatively large cohort of patients consecutively treated with radium-223, in daily practice.

In conclusion, we showed that early ALP dynamics during radium-223 therapy hold potential to serve as a treatmentpredictive biomarker for OS of metastatic CRPC patients in a real-world setting. The findings in this study would benefit from further validation in prospective clinical trials where radium-223 is used according to the current market authorizations.

Funding Open access funding provided by Radboud University Medical Center. This work was supported by the Swedish Cancer Society, the Cancer Society of Stockholm, King Gustav V Jubilee Fund, Stockholm County Council, and Bayer Health Care Sweden and The Netherlands. The funding organizations had no role in the design and conduct of the study, collection, management, analysis, interpretation of the data, and preparation, review, or approval of the manuscript.

\section{Declarations}

Ethical approval and informed consent All procedures performed in this study involving human participants were in accordance with the ethical standards of the institutional and/or national research committees and with the 1964 Helsinki declaration and its later amendments or comparable ethical standards. The study was approved by the local medical ethics committees and both hospital review boards. Informed consent was obtained from all individual participants included in the study.

Conflict of interest MJvdD discloses research grants and travel grants from Bayer, research grants from Janssen-Cilag, and received speaker fees from Astellas. AS declares research funding from Bayer. YM has no conflicts of interest to declare that are relevant to the content of this article. NM reports personal fees from Bayer, grants and personal fees from Jansen-Cilag, personal fees from MSD, grants and personal fees from Roche, grants and personal fees from Astellas, and grants and personal fees from Sanofi. JY has no conflicts of interest to declare that are relevant to the content of this article. WRG is a member of the advisory boards for Bristol-Myers Squibb, Astellas, Janssen, Bayer, Sanofi Genzyme, Amgen, Morphosys and CureVac, has received speaker fees from Bayer and MSD, and has received research funding from Astellas, Bayer, and Janssen. SN has participated as a member of advisory boards for Bayer, Astellas, Janssen, Sanofi Genzyme, and Amgen. IMvO received research grants and personal fees from Astellas, grants and personal fees from Bayer, grants and personal fees from Janssen-Cilag, and grants and personal fees from Sanofi. AU declares research funding from Bayer and Pierre-Fabre and is a member of the advisory boards for PierreFabre, Roche, Pfizer, Merck, Janssen-Cilag, Astellas, and MSD. 
Open Access This article is licensed under a Creative Commons Attribution 4.0 International License, which permits use, sharing, adaptation, distribution and reproduction in any medium or format, as long as you give appropriate credit to the original author(s) and the source, provide a link to the Creative Commons licence, and indicate if changes were made. The images or other third party material in this article are included in the article's Creative Commons licence, unless indicated otherwise in a credit line to the material. If material is not included in the article's Creative Commons licence and your intended use is not permitted by statutory regulation or exceeds the permitted use, you will need to obtain permission directly from the copyright holder. To view a copy of this licence, visit http://creativecommons.org/licenses/by/4.0/.

\section{References}

1. Henriksen G, Breistol K, Bruland OS, Fodstad O, Larsen RH. Significant antitumor effect from bone-seeking, alpha-particleemitting (223)Ra demonstrated in an experimental skeletal metastases model. Cancer Res. 2002;62:3120-5.

2. Parker C, Nilsson S, Heinrich D, Helle SI, O'Sullivan JM, Fossa $\mathrm{SD}$, et al. Alpha emitter radium-223 and survival in metastatic prostate cancer. N Engl J Med. 2013;369:213-23. https://doi.org/10. 1056/NEJMoa1213755.

3. Bubendorf L, Schöpfer A, Wagner U, Sauter G, Moch H, Willi N, et al. Metastatic patterns of prostate cancer: an autopsy study of 1 , 589 patients. Hum Pathol. 2000;31:578-83. https://doi.org/10. 1053/hp.2000.6698.

4. Pezaro CJ, Omlin A, Lorente D, Nava Rodrigues D, Ferraldeschi R, Bianchini D, et al. Visceral disease in castration-resistant prostate cancer. Eur Urol. 2014;65:270-3. https://doi.org/10.1016/j.eururo. 2013.10.055.

5. Heinrich D, Bruland O, Guise TA, Suzuki H, Sartor O. Alkaline phosphatase in metastatic castration-resistant prostate cancer: reassessment of an older biomarker. Future Oncol. 2018;14:2543-56. https://doi.org/10.2217/fon-2018-0087.

6. Brown JE, Cook RJ, Major P, Lipton A, Saad F, Smith M, et al. Bone turnover markers as predictors of skeletal complications in prostate cancer, lung cancer, and other solid tumors. J Natl Cancer Inst. 2005;97:59-69. https://doi.org/10.1093/jnci/dji002.

7. Fizazi K, Massard C, Smith M, Rader M, Brown J, Milecki P, et al. Bone-related parameters are the main prognostic factors for overall survival in men with bone metastases from castration-resistant prostate cancer. Eur Urol. 2015;68:42-50. https://doi.org/10.1016/j. eururo.2014.10.001

8. Saad F, Carles J, Gillessen S, Heidenreich A, Heinrich D, Gratt J, et al. Radium-223 and concomitant therapies in patients with metastatic castration-resistant prostate cancer: an international, early access, open-label, single-arm phase $3 \mathrm{~b}$ trial. Lancet Oncol. 2016;17:1306-16. https://doi.org/10.1016/S1470-2045(16)301735.

9. Sartor O, Coleman RE, Nilsson S, Heinrich D, Helle SI, O’Sullivan $\mathrm{JM}$, et al. An exploratory analysis of alkaline phosphatase, lactate dehydrogenase, and prostate-specific antigen dynamics in the phase 3 ALSYMPCA trial with radium-223. Ann Oncol. 2017;28:10907. https://doi.org/10.1093/annonc/mdx044.

10. Dizdarevic S, Jessop M, Begley P, Main S, Robinson A. (223)RaDichloride in castration-resistant metastatic prostate cancer: improving outcomes and identifying predictors of survival in clinical practice. Eur J Nucl Med Mol Imaging. 2018;45:2264-73. https:// doi.org/10.1007/s00259-018-4083-3.

11. Van der Doelen MJ, Mehra N, Hermsen R, Janssen MJR, Gerritsen WR, Van Oort IM. Patient selection for radium-223 therapy in patients with bone metastatic castration-resistant prostate cancer: new recommendations and future perspectives. Clin Genitourin Cancer. 2019;17:79-87. https://doi.org/10.1016/j.clgc.2018.11. 008.

12. Van der Doelen MJ, Kuppen MCP, Jonker MA, Mehra N, Janssen MJR, van Oort IM, et al. 223Ra therapy in patients with advanced castration-resistant prostate cancer with bone metastases: lessons from daily practice. Clin Nucl Med. 2018;43:9-16. https://doi.org/ 10.1097/RLU.0000000000001904.

13. Alva A, Nordquist L, Daignault S, George S, Ramos J, Albany C, et al. Clinical correlates of benefit from radium-223 therapy in metastatic castration resistant prostate cancer. Prostate. 2017;77:47988. https://doi.org/10.1002/pros.23286.

14. Wong WW, Anderson EM, Mohammadi H, Daniels TB, Schild SE, Keole SR, et al. Factors associated with survival following radium223 treatment for metastatic castration-resistant prostate cancer. Clin Genitourin Cancer. 2017;15:e969-e75. https://doi.org/10. 1016/j.clgc.2017.04.016.

15. Sartor O, Coleman R, Nilsson S, Heinrich D, Helle SI, O'Sullivan $\mathrm{JM}$, et al. Effect of radium-223 dichloride on symptomatic skeletal events in patients with castration-resistant prostate cancer and bone metastases: results from a phase 3, double-blind, randomised trial. Lancet Oncol. 2014;15:738-46. https://doi.org/10.1016/S14702045(14)70183-4.

16. Cornford P, Bellmunt J, Bolla M, Briers E, De Santis M, Gross T, et al. EAU-ESTRO-SIOG guidelines on prostate cancer. Part II: treatment of relapsing, metastatic, and castration-resistant prostate cancer. Eur Urol. 2017;71:630-42. https://doi.org/10.1016/j. eururo.2016.08.002.

17. Scher HI, Morris MJ, Stadler WM, Higano C, Basch E, Fizazi K, et al. Trial design and objectives for castration-resistant prostate cancer: updated recommendations from the prostate cancer clinical trials working group 3. J Clin Oncol. 2016;34:1402-18. https://doi. org/10.1200/JCO.2015.64.2702.

18. Chi KN, Kheoh T, Ryan CJ, Molina A, Bellmunt J, Vogelzang NJ, et al. A prognostic index model for predicting overall survival in patients with metastatic castration-resistant prostate cancer treated with abiraterone acetate after docetaxel. Ann Oncol. 2016;27:454 60. https://doi.org/10.1093/annonc/mdv594.

19. Armstrong AJ, Garrett-Mayer E, de Wit R, Tannock I, Eisenberger M. Prediction of survival following first-line chemotherapy in men with castration-resistant metastatic prostate cancer. Clin Cancer Res. 2010;16:203-11. https://doi.org/10.1158/1078-0432.Ccr-092514.

20. McKay RR, Jacobus S, Fiorillo M, Ledet EM, Cotogna PM, Steinberger AE, et al. Radium-223 use in clinical practice and variables associated with completion of therapy. Clin Genitourin Cancer. 2017;15:e289-e98. https://doi.org/10.1016/j.clgc.2016. 08.015 .

21. Saad F, Gillessen S, Heinrich D, Keizman D, O'Sullivan JM, Nilsson S, et al. Disease characteristics and completion of treatment in patients with metastatic castration-resistant prostate cancer treated with radium-223 in an international early access program. Clin Genitourin Cancer. 2019;17:348-55 e5. https://doi.org/10.1016/j. clgc.2019.05.012.

22. O'Sullivan JM, Carles J, Cathomas R, Gomez-Iturriaga A, Heinrich D, Kramer G, et al. Radium-223 within the evolving treatment options for metastatic castration-resistant prostate cancer: recommendations from a European Expert Working Group. Eur Urol Oncol. 2020;3:455-63. https://doi.org/10.1016/j.euo.2019.02.007.

23. Kuppen MCP, Westgeest HM, van der Doelen MJ, van den Eertwegh AJ, Coenen JL, Aben KK, et al. Real-world outcomes of radium-223 dichloride for metastatic castration resistant prostate cancer. Future Oncol. 2020;16:1371-84. https://doi.org/10.2217/ fon-2020-0039. 
24. Sternberg CN, Tombal B, Miller K, Saad F, Sartor O, Sade JP, et al. Use of bone health agents (BHAs) in patients (pts) with metastatic castration-resistant prostate cancer (mCRPC) treated with radium223 (Ra-223) after abiraterone (Abi): an interim review of REASSURE. Ann Oncol. 2018;29:viii288. https://doi.org/10. 1093/annonc/mdy284.035.

25. Smith M, Parker C, Saad F, Miller K, Tombal B, Ng QS, et al. Addition of radium-223 to abiraterone acetate and prednisone or prednisolone in patients with castration-resistant prostate cancer and bone metastases (ERA 223): a randomised, double-blind, placebo-controlled, phase 3 trial. Lancet Oncol. 2019;20:408-19. https://doi.org/10.1016/S1470-2045(18)30860-X.

26. Caffo O, Frantellizzi V, Tucci M, Galli L, Monari F, Baldari S, et al. Fracture risk and survival outcomes in metastatic castrationresistant prostate cancer patients sequentially treated with abiraterone acetate and radium-223. Eur J Nucl Med Mol Imaging. 2020;47:2633-8. https://doi.org/10.1007/s00259-02004796-w.
27. Agarwal N, Nussenzveig R, Hahn AW, Hoffman JM, Morton K, Gupta S, et al. Prospective evaluation of bone metabolic markers as surrogate markers of response to radium-223 therapy in metastatic castration-resistant prostate cancer. Clin Cancer Res. 2020;26: 2104-10. https://doi.org/10.1158/1078-0432.Ccr-19-2591.

28. Nilsson S, Franzen L, Parker C, Tyrrell C, Blom R, Tennvall J, et al. Bone-targeted radium-223 in symptomatic, hormone-refractory prostate cancer: a randomised, multicentre, placebo-controlled phase II study. Lancet Oncol. 2007;8:587-94. https://doi.org/10. 1016/S1470-2045(07)70147-X.

29. Suominen MI, Fagerlund KM, Rissanen JP, Konkol YM, Morko JP, Peng Z, et al. Radium-223 inhibits osseous prostate cancer growth by dual targeting of cancer cells and bone microenvironment in mouse models. Clin Cancer Res. 2017;23:4335-46. https://doi.org/10.1158/ 1078-0432.CCR-16-2955.

Publisher's note Springer Nature remains neutral with regard to jurisdictional claims in published maps and institutional affiliations. 